\title{
O USO DE SISTEMAS EMBARCADOS PARA IDENTIFICAR O ESTRESSE AO CALOR EM VACAS LEITEIRAS
}

Larissa Christyna de Paula; João Paulo Barros; João Victor Firmino Garcia; Aline Sousa Camargos; Paulo Eduardo Nogueira; Eliandra Maria Bianchini Oliveira; Wallacy Barbacena Rosa dos Santos; Fernando Matos.

Instituto Federal de Educação, Ciência e Tecnologia Goiano, Campus Morrinhos. E-mail: larissachristynadepaula@gmail.com

\section{RESUMO}

Os sistemas embarcados estão presentes no cotidiano dos seres humanos, desde um simples micro-ondas a um carro dos anos 2000. Nos últimos anos esses sistemas têm se disseminado na agropecuária brasileira, como por exemplo, controle de irrigação e controle de alimentação de animais. A tolerância ao calor e a adaptabilidade a ambientes tropicais são fatores importantes a serem considerados na bovinocultura leiteira. O aumento da temperatura ambiente e, consequentemente, do estresse ao calor provoca uma série de efeitos no metabolismo do animal que alteram o seu comportamento e o bem-estar, afetando diretamente a produção de leite. A temperatura superficial e a frequência respiratória são variáveis que podem ser utilizadas para identificar o estresse ao calor pelos animais. Assim, um dispositivo, utilizando a plataforma Arduíno, será desenvolvido para medir a temperatura superficial e a frequência respiratória do animal, permitindo uma maior confiabilidade nas informações geradas.

Palavras-chave: adaptabilidade; bioclimatologia; bovinocultura leiteira; clima tropical; tecnologia de precisão.

\section{THE USE OF BOATS TO IDENTIFY HEAT STRESS IN DAIRY COWS}

\begin{abstract}
The embedded systems are present in the daily life of humans, from a simple microwave to a car of the years 2000. In recent years these systems have spread in Brazilian agriculture, such as irrigation control and animal feed control. Heat tolerance and adaptability to tropical environments are important factors to be considered in dairy cattle. The increase in the ambient temperature and, consequently, the heat stress causes a series of effects on the animal's metabolism that alter its behavior and well-being, directly affecting milk production. Surface temperature and respiratory rate are variables that can be used to identify heat stress by animals. Thus, a device, using the Arduino platform, it will be developed to measure the surface temperature and the respiratory rate of the animal, allowing a greater reliability in the information generated.
\end{abstract}

Keywords: adaptability; bioclimatology; dairy cattle; tropical weather; precision technology.

\section{INTRODUÇÃO}

Nas regiões tropicais, durante a maior parte do ano, a temperatura do ar combinada a outros parâmetros ambientais, pode provocar estresse ao calor nos animais. Estes buscam se ajustar aumentando a dissipação de calor por meio, principalmente, pela termólise cutânea e da 
respiratória (Silva, 2000). Sendo assim, os sistemas de produção devem identificar corretamente quais os fatores climáticos que influenciam negativamente a bovinocultura leiteira como, por exemplo, o estresse ao calor causado pelo ambiente.

Segundo Baccari Júnior et al. (1993), a avaliação da relação básica entre os animais e seu ambiente térmico começa com a zona de termoneutralidade, que é a faixa de temperatura ambiente efetiva dentro da qual o custo fisiológico é mínimo e a retenção energética da dieta é máxima e o desempenho produtivo esperado são máximos. De acordo com o mesmo autor, a zona de termoneutralidade define limites de temperatura, que são as temperaturas crítica superior e inferior.

De acordo com Santos et al. (2006), se a temperatura do ar aumentar, diminui a perda de calor de forma sensível, aumentando a temperatura do núcleo central e desta forma o organismo do animal, através de mecanismos evaporativos como a sudorese e/ou frequência respiratória, aumenta a dissipação de calor insensível.

Nas regiões de clima tropical, o estresse térmico pelo calor é um dos principais fatores que limitam o desenvolvimento dos animais. As regiões tropicais posem ser caracterizadas por altos níveis de radiação solar e temperatura que podem afetar negativamente a produção animal (McManus et al., 2009).

A alta incidência de radiação solar e as altas temperaturas impõem durante praticamente todos os meses do ano, situações de desconforto térmico aos animais, sobretudo aos de raças mais produtivas, que geralmente são oriundas de clima temperado (Souza Junior et al., 2008). Animais criados com variações climáticas fora da faixa de conforto térmico podem apresentar perda de peso, crescimento retardado, problemas respiratórios e hormonais, falta de apetite e baixo rendimento de carcaça. Sendo assim, o efeito do clima sobre o desenvolvimento dos animais de produção tem despertado atenção, salientando a importância da interação animal-ambiente como fator relevante aos processos produtivos (Souza Junior et al., 2004).

Portanto, é de fundamental importância detectar os agentes causadores de estresse ao calor em animais de produção leiteira, auxiliando ao produtor rural as soluções eficientes para minimizar esses efeitos em sua propriedade, podendo assim conciliar a uma produção leiteira com o conforto térmico e o bem-estar animal.

Desta forma, o desenvolvimento de novas tecnologias que mensuram a temperatura superficial e a frequência respiratória do animal por meio de sistemas embarcados, como sensores, irá facilitar e agilizar a identificação de estresse pelo calor dos animais como também uma maior confiabilidade das informações obtidas.

Objetivou-se com essa revisão de literatura elencar alguns pontos da utilização da ferramenta de sistemas embarcados e do estresse ao calor em vacas leiteiras.

\section{ESTRESSE AO CALOR E AS VÁRIAVEIS FISIOLOGICAS: FREQUÊNCIA RESPIRATORIA E TEMPERATURA RETAL}

O estresse pode ser entendido como uma resposta coordenada do organismo a agentes estressores que leva à secreção de vários hormônios e causa alterações no comportamento e nas funções orgânicas (CARRASCO \& VAN DE KAR, 2003).

Mudanças no ambiente podem levar os animais a desequilíbrios com o meio e esta resposta ocorre de forma individual. Desta forma, a caracterização do clima ideal para os animais domésticos e o estudo das reações ao estresse térmico pelo calor devem ser identificados para que se possa indicar a melhor prática de manejo, modelo adequado de instalações e plano nutricional, afim de que, os animais expressem suas aptidões zootécnicas (Oliveira et al., 2005).

Souza et al. (2010) reportam que nos últimos anos, com as mudanças climáticas, o número de pesquisas buscando o bem-estar animal têm se intensificado na tentativa de minimizar as perdas econômicas decorrentes dos efeitos do clima sobre a produção animal nos trópicos. 0 
bem-estar animal pode ser considerado uma demanda para qualquer sistema criatório que deseja ser eticamente defensável e socialmente aceitável (Ferreira et al., 2011).

Por conta desse cenário, deve-se levar em consideração algumas variáveis, entre elas: o comportamento maternal, o comportamento de pastejo, resistência a doenças, reatividade comportamental, longevidade, sucesso reprodutivo, mortalidade, desafios nutricionais e térmicos, dor, liberdade comportamental, genética e o ambiente em que o animal está inserido (Wolf et al., 2008). Os princípios fundamentam-se nos conceitos das cinco liberdades inicialmente propostos pelo conselho de bem-estar de animais de produção e modificado por Molento (2006) sendo eles: (1) liberdade nutricional, (2) liberdade sanitária, (3) liberdade ambiental, (4) liberdade comportamental e (5) liberdade psicológica.

O principal objetivo dessas regras são o de garantir que esses animais possam ter ao longo de sua vida uma criação mais digna e/ou humanizada. Porém muitas vezes produtores não percebem que além de estarem garantindo melhores condições de vida para seus animais, eles também lhes garantirão melhores resultados produtivos que podem ser vistos como bonificações por meio desse melhor método de criação de seus rebanhos.

Animais criados em ambientes onde ficam susceptíveis a quaisquer tipos de estresse terão consequências negativas relacionadas ao seu rendimento produtivo. Brown-Brandl (2005) e Tratcher (1974) observaram que animais criados em ambientes onde ficam susceptíveis a faixas de variações climáticas fora da zona de conforto térmico, apresentarão respostas negativas, tais como, redução da ingestão de alimentos, ineficiência em seu crescimento, baixa produtividade, perda de peso, e dificuldade respiratória.

O estresse térmico pode ser mensurado através das variáveis fisiológicas, tais como temperatura retal, frequência respiratória, perfil hematológico e hormonal. Do ponto de vista bioclimático, mesmo para animais considerados tolerantes ao calor, podem ocorrer alterações comportamentais e fisiológicas (Brown-Brandl et al., 2003; Ferreira et al., 2006).

Tanto a temperatura retal, como a frequência respiratória, sofrem influências intrínsecas, como exercícios físicos, medo, excitação, estado fisiológico e produção de leite, e extrínsecas, como temperatura e umidade do ar, radiação solar, velocidade dos ventos, estação do ano, hora do dia e sombreamento. Em situações de estresse térmico, a frequência respiratória se eleva, antes do aumento da temperatura retal (Ferreira et al., 2006).

Animais submetidos ao estresse térmico consomem mais água, provavelmente, para compensar o aumento do potencial de perda por evaporação, através da elevação da frequência respiratória (Ferreira et al., 2006; Al-Tamimi, 2007). De acordo com Cunningham (2004), vários fatores são capazes de causar variações na temperatura corporal, entre os quais: idade, sexo, estação do ano, período do dia, exercício, ingestão e digestão de alimentos.

\section{ZONA DE CONFORTO TÉRMICO}

Para evitar esse desgaste excessivo fisiológico é fundamental oferecer aos animais, ambientes de criação ao qual os mesmos estarão adaptados, onde eles se encontrarão dentro de suas zonas de conforto térmico. BACCARI JUNIOR (1998) descreve a zona de conforto termino ou zona de termoneutralidade, como o ambiente de criação onde os animais não necessitariam de ativar seus mecanismos termorreguladores, tanto para capturar como para dissipar calor, tendo um gasto de energia de manutenção mínimo, podendo usar assim essa energia para um melhor potencial de desempenho produtivo.

A zona de conforto térmico varia entre as espécies de animais e até mesmo entre os bovinos, essa variação de temperatura pode ser bem expressiva dependendo do grupo de origem desses animais. A origem dos bovinos pode ser dividida em dois grandes grupos, as raças de origem europeias e as raças de origem indiana. A zona de conforto térmico do grupo dos taurinos, é de $0^{\circ} \mathrm{C}$ a $16^{\circ} \mathrm{C}$, já o grande grupo das raças zebuínas possui uma zona de conforto térmico mais 
elevada, podendo variar de $10^{\circ} \mathrm{C}$ a $27^{\circ} \mathrm{C}$, devido a sua adaptabilidade a regiões mais tropicais (Carvalho et al., 1995).

Segundo Nääs (1998) como média geral a maioria dos ruminantes podem sobreviver confortavelmente entre uma faixa de temperatura entre $13^{\circ} \mathrm{C}$ e $18^{\circ} \mathrm{C}$. Já para as vacas em lactação essa zona termina possui uma maior amplitude, tal qual seria entre $4^{\circ} \mathrm{C}$ e $26^{\circ} \mathrm{C}$, com limites expressos entre $7^{\circ} \mathrm{C}$ e $21^{\circ} \mathrm{C}$ em função do ambiente.

Certas diferenças entre as zonas de conforto térmico dos dois grandes grupos dos bovinos são devidas as suas diferenciações evolutivas, que garantiram características fisiológicas e anatômicas diferenciadas de acordo com o ambiente onde foram criados para lhes garantir uma boa adaptabilidade. Pan (1963) cita algumas dessas diferenças, como diferentes tamanhos de superfície corporal, membros mais longos, melhor capacidade de sudação e irrigação vascular, diferenciação gandular da pele, e a variação da cor e tipo de pelame.

Quando submetidos a uma zona de estresse ao calor os bovinos desempenha um gasto fisiológico muito maior do que quando se encontra dentro da zona de termoneutralidade, esse gasto fisiológico é um preço que os animais não conseguem sustentar por muito tempo. Para conseguirem se libertarem logo dessa zona de desconforto térmico os animais buscam por si só meios de contornar essa situação, utilizando-se de técnicas de troca de calor.

Os bovinos por serem animais homeotérmicos conseguem controlar sua temperatura corporal até certo ponto de acordo com a variação climática do ambiente. Essa dissipação de calor é realizada principalmente por meio da termólise cutânea e respiratória (Silva, 2000). Os mecanismos de perda de calor podem classificados em tipos diferentes, Silva (2000) cita como um deles a perda de calor sensível, que pode se dar por radiação, condução e convecção, tendo também a perda de insensível, através da evaporação, ou seja, sudação e respiração.

Quando o animal está no estado de estresse ao calor os mecanismos de perda de calor sensíveis são os primeiros a serem acionados, para que ocorra uma boa funcionalidade dos mesmos, se faz necessário uma baixa temperatura do ar em relação a temperatura corporal do animal. Se os mecanismos de perda de calor sensíveis não estiverem tendo uma funcionalidade tão efetiva, logo em seguida os mecanismos de perda de calor insensíveis serão ativados devido ao aumento da temperatura do núcleo central do animal, assim acarretando o acionamento imediato dos mecanismos evaporativos de perda de calor, ocorrendo então a dissipação do calor (Santos et al, 2006).

\section{TESTES DE ADAPTABILIDADE}

Quando animais ficam submetidos a estados de estresse calórico, suas funções fisiológicas começam a desencadear alterações na tentativa de desencadear um balanço térmico para promover uma homeoterma corporal. As distintas respostas fisiológicas na tentativa de alcançar seu equilíbrio térmico irão variar de acordo com a espécie, raça, idade, sexo estado fisiológico, e variáveis ambientais, onde podemos destacar a umidade relativa do ar, temperatura e hora do dia (BIRGEL JUNIOR, 2001).

As respostas fisiológicas que são desencadeias por diferentes indivíduos sobre o estado de estresse ao calor, indica a capacidade fisiológica de que o animal possui de tolerar e dissipar esse calor. Existem alguns métodos que podem ser utilizados para avaliar o nível de tolerância de estresse calórico que o animal poderá vir a tolerar, esses testes ficaram conhecidos como, índice de tolerância ao calor (ITC) (VERISSIMO, 2009).

O ITC é um determinante para informar se o animal avaliado está ou não é um estado de estresse calórico, por esse motivo aconselha-se sempre a utilização de algum outro teste juntamente com ele, para que além de descobrirmos se o animal está ou não com alterações em seu estado fisiológico, também possamos averiguar o grau de sofrimento do animal (De Souza, 2010). Segundo PAES (2000) um dos testes mais utilizados juntamente com o de ITC, é o 
hemograma, pois ele indica com eficiência o estado de saúde do animal e grau do nível de estresse.

Por conta da necessidade de se constatar o índice de tolerância ao calor, vários testes foram desenvolvidos entre eles:

1) Teste de Benezra: Segundo Müller (1982) o teste de Benezra consiste em um teste de adaptabilidade, que se utiliza da seguinte formula para realizar o cálculo do coeficiente de tolerância ao calor $(\mathrm{CTC})$. CTC $=(T R / 39,5)+(F R / 25)=2$, onde quanto mais próximo de 2 for o resultado obtido, amais adaptado ao calor o animal estará, sendo que $\mathrm{TC}=$ temperatura retal ou corporal medida em ${ }^{\circ} \mathrm{C}$; $\mathrm{FR}=$ frequência respiratória, em movimentos por minuto. A formula mencionada pode ser utilizada por um amplo grupo de animais, como por exemplo para bovinos, caprinos e ovinos, desde que sobram adaptações necessárias de acordo com os dados fisiológicos normais de cada espécie (KOLB, 1984).

2) Teste de Rauschenbach - Yerokhin: 0 teste de Rauschenbach e Yerokhin consiste na seguinte formula: ITC $=2\left(0,5 t_{2}-10 d t+30\right)$, onde: ITC = índice de tolerância ao calor; $t_{2}=$ temperatura do ar no período da tarde; $d t=$ diferença entre a temperatura corporal à tarde e a temperatura corporal da manhã. O animal apresentará maior nível de adaptabilidade, quanto mais próximo de 100 for o resultado obtido (Ferreira, 2005).

3) Teste de Ibéria: $O$ teste de ibéria utiliza a seguinte formula: $C T C=100-[18(T R-38,3)]$. $\mathrm{CTC}=$ coeficiente de tolerância ao calor; 100 = eficiência máxima em manter a temperatura corporal em $39,1^{\circ} \mathrm{C} ; 18=$ constante; $\mathrm{TR}=$ temperatura retal media final; $38,3^{\circ} \mathrm{C}=$ temperatura corporal normal. A adaptabilidade dos bovinos é medida em relação a sua exposição ao sol, durante a aplicação do teste devem ser feitas aferições diárias, por três dias consecutivos ou não, onde deve-se registar a TR (temperatura retal) as 10:00 e as 15:00 horas, cujos valores serão usados para calcular a temperatura retal media final. Quanto mais próximo de 100, mais adaptado o animal estará ao ambiente (Müller, 1982).

4) Teste de Baccari Júnior: o teste de Baccari Júnior avalia a índice de tolerância ao calor dos animais, por meio da exposição dos mesmos ao sol, aferindo sua temperatura no momento de exposição à radiação solar e observando em seguida a diminuição da temperatura corporal dos animais até níveis considerados de condições naturais do ambiente. A realização do teste ocorre da seguinte maneira. Os animais utilizados no teste devem ser mantidos duas horas em um ambiente com sombreamento (ao meio dia); em seguida por uma hora em exposição direta a radiação solar, sendo que nesse período de exposição que será coletada pela primeira vez a temperatura retal (TR1); posteriormente serão conduzidos para a área sombreada, mantendo os animais em total estado de repouso por uma hora, para em seguida ser coletada a (TR2), onde posteriormente serão utilizadas no cálculo do ITC, utilizando a seguinte formula: ITC = $10-($ TR2 - TR1). ITC = índice de tolerância ao calor; 10 = constante; TR1 = temperatura retal no momento da exposição ao sol; TR2 = temperatura retal no momento de repouso. Sendo que quando mais próximo o resultado for de 10, mais adaptado o animal estará ao ambiente (Baccari Jr, 1990).

\section{SISTEMAS EMBARCADOS}

Os processos de informatização e tecnificação vem se tornando algo inevitável a medida que avançamos no tempo. $O$ uso de tecnologias está se tornando cada vez mais comum no nosso dia-a-dia, e sempre cada vez mais atrativo através de suas surpreendentes promessas. Adicionar tecnologia as tarefas de nosso cotidiano já se tornaram algo tendencioso nos dias de hoje.

Um belo exemplo é o simples uso de um micro-ondas, que consegue com uma tecla camuflar toda a operação computacional existente que teria por trás, por exemplo, no processo de se estourar uma pipoca, onde todo o sistema tem que considerar inúmeras variáveis, como sua potência, tempo, acionamento de motor, permissão de interrupção, entre outras (Cunha, 2007). 
Em relação a sua composição, geralmente todos os sistemas embarcados devem possuir uma unidade de processamento, que é a parte responsável por realizar as instruções de um programa. Esses sistemas, também são compostos por um software, que seria um conjunto de programas embarcado na unidade de processamento, o qual foi escrito para realizar operações específicas a qual foi proposto (Ball, 2005).

No momento de elaboração de um sistema embarcado existem algumas características que devem ser levadas em conta. Sua composição de peso e tamanho sempre devem ser as menores possíveis, pois essas características são fatores determinantes para o sucesso e popularização do produto. Além disso, a eficiência energética dos sistemas deve ter tamanho cuidado, pois quanto menor o consumo de energia, maior será a sua autonomia, implicando assim, na competitividade do produto (Cunha, 2007).

Em relação ao acompanhamento do estresse animal, esses sistemas oferecem dispositivos para a captura de dados com a mínima intervenção humana possível. Através de sensores é possível coletar a temperatura e a pulsação animal. Esses sensores estão conectados a um circuito, que de posse dos dados de temperatura e pulsação, pode realizar os cálculos necessários e indicar o nível de estresse do animal ou, através de um painel visual, também acoplado a placa, também podendo ser através de uma mensagem enviada ao celular do produtor.

As inovações tecnológicas desenvolvidas pela zootecnia de precisão têm como objetivo principal melhorar o ambiente de vivencia dos animais, sua produtividade, crescimento, melhorar suas condições zootécnicas, que envolvem também o bom manejo e uma boa sanidade dos sistemas de produção (Frost, 1997).

As formais comuns onde vem se aplicando tecnologias, são na identificação e pesagem dos animais, no transporte e monitoramento de funções fisiológicas, principalmente para averiguar temperatura corporal, taxa respiratória e cardíaca. Bandeira Filho (2003) destaca o uso de equipamentos para monitorar a medida de parâmetros ambientais, tais como temperatura e umidade, podendo também ser utilizadas como ferramentas de registro de vacinação, cio, parto, etc. Sendo que tais averiguações podem ser feitas por equipamentos ligados a sistemas coletores de dados, e mais atualmente a análise de imagens (XIN; SHAO, 2002).

\section{CONSIDERAÇÕES FINAIS}

Portanto a utilização de novas tecnologias embarcadas como os sensores para avaliar a temperatura superficial e a frequência respiratória podem favorecer uma maior confiabilidade das pesquisas de tolerância ao calor e bem-estar animal. Buscando sempre maximizar seus resultados, através da utilização dessas novas ferramentas empregadas na produção animal, assim facilitando a aquisição de dados para análises mais apuradas, contribuindo para o avanço e velocidade das pesquisas.

\section{REFERÊNCIAS}

ALVES, Sulivan Pereira. Uso da zootecnia de precisão na avaliação do bem-estar bioclimático de aves poedeiras em diferentes sistemas de criação. 2006. Tese de Doutorado. Escola Superior de Agricultura" Luiz de Queiroz.

BACCARI JÚNIOR, F. Métodos e técnicas de avaliação da adaptabilidade dos animais às condições tropicais. Simpósio Internacional de Bioclimatologia Animal nos Trópicos: pequenos e grandes ruminantes, v. 1, p. 9-17, 1990.

BIRGEL JÚNIOR, E. H. et al. Valores de referência do eritrograma de bovinos da raça Jersey criados no Estado de São Paulo. Arquivo Brasileiro de Medicina Veterinária e Zootecnia, 2001. https://doi.org/10.1590/S0102-09352001000200006 
CHASE, Otavio; ALMEIDA, F. J. Sistemas embarcados. Mídia Eletrônica. Página na internet:< www. sbajovem. org/chase>, capturado em, v. 10, n. 11, 2007.

DA COSTA FERRO, Diogo Alves. EFEITO dOS ELEMENTOS CLIMÁtICOS NA PRODUÇÃO E REPRODUÇÃO DE VACAS LEITEIRAS.

DAMASCENO, Júlio Cesar et al. Respostas comportamentais de vacas holandesas, com acesso à sombra constante ou limitada. Pesquisa Agropecuária Brasileira, v. 34, n. 4, p. 709-715, 1999.

DA NÓBREGA, Giovanna Henriques et al. A produção animal sob a influência do ambiente nas condições do semiárido nordestino. Revista verde de agroecologia e desenvolvimento sustentável, v. 6, n. 1, p. 67-73, 2011.

DE SOUSA JÚNIOR, Severino Cavalcante et al. Características termorreguladoras de caprinos, ovinos e bovinos em diferentes épocas do ano em região semi-árida. Revista Científica de Produção Animal, v. 10, n. 2, 2008.

DE SOUZA, Bonifácio Benício et al. Efeito do ambiente sobre as respostas fisiológicas de Caprinos saanen e mestiços $1 / 2$ saanen+ $1 / 2$ boer no semi-árido Paraibano. Agropecuária científica no semiárido, v. 6, n. 2, 2010.

FERREIRA, Rony Antonio. Maior produção com melhor ambiente para aves, suínos e bovinos. Aprenda Fácil, 2005.

GERVINI, Alexandre I. et al. Avaliação de Desempenho, Área e Potência de Mecanismos de Comunicação em Sistemas Embarcados. SEMISH'03-XXX Seminário Integrado de Software e Hardware, 2003.

KOLB, E.; KOLB, E. Fisiologia da absorção e da digestão. KOLB, E. Fisiologia veterinária, v. 4, p. 105207, 1984.

MOLENTO, Carla Forte Maiolino. Repensando as cinco liberdades. In: CONGRESSO INTERNACIONAL CONCEITOS EM BEMESTAR ANIMAL. 2006.

MÜLLER, Pedro Bernardo. Bioclimatologia, aplicada aos animais domésticos. 1982.

PAES, P. R. et al. Comparação dos valores hematológicos entre caprinos fêmeas da raça Parda Alpina de diferentes faixas etárias. Veterinária Notícias, v. 6, n. 1, p. 43-49, 2000.

PANDORFI, Héliton; ALMEIDA, Gledson Luiz Pontes; GUISELINI, Cristiane. Zootecnia de precisão: princípios básicos e atualidades na suinocultura. Revista Brasileira de Saúde e Produção Animal, v. 13, n. 2, p. 558-568, 2012. https://doi.org/10.1590/S1519-99402012000200023

ROBERTO, João Vinícius Barbosa et al. Gradientes térmicos e respostas fisiológicas de caprinos no semiárido brasileiro utilizando a termografia infravermelha. Journal of Animal Behavior and Biometeorology, v. 2, n. 1, p. 11-19, 2014. https://doi.org/10.14269/2318-1265.v02n01a03 
SANTOS, Jose Romulo Soares dos et al. Respostas fisiológicas e gradientes térmicos de ovinos das raças Santa Inês, Morada Nova e de seus cruzamentos com a raça Dorper às condições do semiárido nordestino. Ciência e Agrotecnologia, 2006.

SEVEGNANI, Kelly B. et al. Zootecnia de precisão: análise de imagens no estudo do comportamento de frangos de corte em estresse térmico. Revista Brasileira de Engenharia Agrícola e Ambiental, v. 9, n. 1, p. 115-119, 2005.

SILVA, Iran José Oliveira da USP; PANDORFI, Héliton USP; PIEDADE, Sônia Maria Stefano. Uso da zootecnia de precisão na avaliação do comportamento de leitões lactentes submetidos a diferentes sistemas de aquecimento. Revista Brasileira de Zootecnia, 2005.

SILVA, R. G. Introdução a bioclimatologia animal. São Paulo: Nobel, 286p, 2000.

VERÍSSIMO, Cecília José et al. Tolerância ao calor em ovelhas Santa Inês de pelagem clara e escura. Revista Brasileira de Saúde e Produção Animal, v. 10, n. 1, p. 159-167, 2009.

MOLENTO, C. F. M. Repensando as cinco liberdades. Curitiba, 2006a. Disponível em: . Acesso em: 19 set. 2013. MOLENTO, C. F. M. Senciência animal. Curitiba: Conselho Regional de Medicina

Wolf, B. T., McBride, S. D., Lewis, R. M., Davies, M. H. \& Haresign, W. 2008. Estimates of the genetic parameters and repeatability of behavioural traits of sheep in an arena test. Applied Animal Behaviour Science, 112, 68- 80. https://doi.org/10.1016/j.applanim.2007.07.011 